Supporting Information

\title{
Structure, Molecular Interactions, and Dynamics of Aqueous [BMIM][BF 4 Mixtures: A Molecular Dynamics Study
}

Tsun-Mei Chang* and Stephanie E. Billeck

Department of Chemistry

University of Wisconsin-Parkside

Kenosha, WI 53141

*Email: chang@uwp.edu 
The potential energy function for the polarizable forcefield is expressed as

$$
\begin{aligned}
U= & \sum_{\text {bonds }} K_{r}\left(r-r_{e q}\right)^{2}+\sum_{\text {angles }} K_{\theta}\left(\theta-\theta_{e q}\right)^{2}+\sum_{\text {dihedrals }} \frac{V_{n}}{2}[1+\cos (n \varphi-\gamma)] \\
& +\sum_{i} \sum_{j>i}\left(4 \varepsilon_{i j}\left[\left(\frac{\sigma_{i j}}{r_{i j}}\right)^{12}-\left(\frac{\sigma_{i j}}{r_{i j}}\right)^{6}\right]+\frac{1}{4 \pi \varepsilon_{o}} \frac{q_{i} q_{j}}{r_{i j}}\right)-\frac{1}{2} \sum_{i} \alpha_{i} \boldsymbol{E}_{i}^{0} \cdot \boldsymbol{E}_{i} .
\end{aligned}
$$

Here $\boldsymbol{E}_{i}^{0}$ is the electric field at site $i$ due to the fixed charges in the system and $\boldsymbol{E}_{i}$ is the total electric field at site $i$.

$$
\boldsymbol{E}_{i}^{0}=\sum_{j \neq i} \frac{q_{j} \boldsymbol{r}_{i j}}{r_{i j}^{3}} \quad \boldsymbol{E}_{i}=\boldsymbol{E}_{i}^{0}+\sum_{j \neq i} \boldsymbol{T}_{i j} \cdot \alpha_{i} \boldsymbol{E}_{i} \quad \boldsymbol{T}_{i j}=\frac{1}{r_{i j}^{3}}\left(\frac{3 \boldsymbol{r}_{i j} \boldsymbol{r}_{i j}}{r_{i j}^{2}}-1\right)
$$

The partial charges (in unit of electron) for $[\mathrm{bmim}]^{+},\left[\mathrm{BF}_{4}\right]^{-}$, and $\mathrm{H}_{2} \mathrm{O}$ are shown in the following figures.
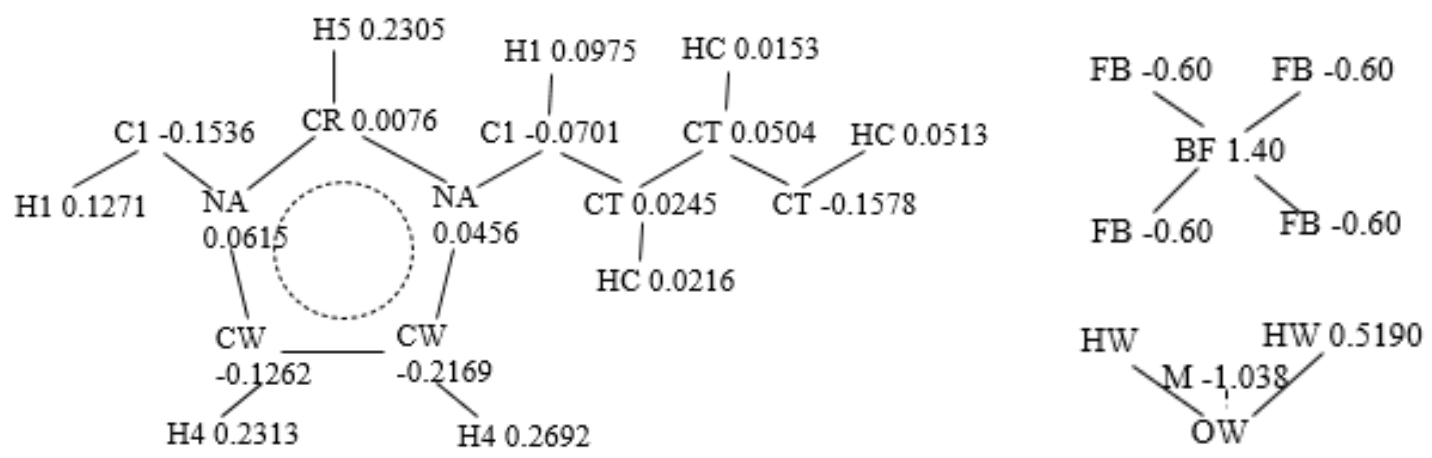

The forcefield parameters are listed in the tables below.

Table S1: Lennard-Jones parameters and point polarizabilities.

\begin{tabular}{|c|c|c|c|c|c|c|c|}
\hline Atom type & $\sigma(\AA)$ & $\varepsilon(\mathrm{kcal} / \mathrm{mol})$ & $\alpha\left(\AA^{3}\right)$ & Atom type & $\sigma(\AA)$ & $\varepsilon(\mathrm{kcal} / \mathrm{mol})$ & $\alpha\left(\AA^{3}\right)$ \\
\hline $\mathrm{C} 1$ & 3.296 & 0.1150 & 0.878 & $\mathrm{HC}$ & 2.650 & 0.0157 & 0.135 \\
$\mathrm{CW}$ & 3.332 & 0.1100 & 0.360 & $\mathrm{NA}$ & 3.136 & 0.1900 & 0.530 \\
$\mathrm{CR}$ & 3.332 & 0.1100 & 0.360 & $\mathrm{BF}$ & 3.428 & 0.2000 & 0.500 \\
$\mathrm{CT}$ & 3.400 & 0.1094 & 0.878 & $\mathrm{FB}$ & 3.428 & 0.1000 & 0.610 \\
$\mathrm{H} 1$ & 1.960 & 0.0300 & 0.135 & $\mathrm{HW}$ & 1.782 & 0.0000 & 0.000 \\
$\mathrm{H} 4$ & 2.049 & 0.0260 & 0.167 & $\mathrm{OW}$ & 3.221 & 0.1825 & 0.000 \\
$\mathrm{H} 5$ & 2.049 & 0.0300 & 0.167 & $\mathrm{M}$ & & & 1.444 \\
\hline
\end{tabular}

Table S2: Bond parameters.

\begin{tabular}{|c|c|c|c|c|c|}
\hline Bond & $\mathrm{r}_{\mathrm{eq}}(\AA)$ & $\mathrm{K}_{\mathrm{r}}\left(\mathrm{kcal} / \mathrm{mol}^{\circ} \AA^{2}\right)$ & Bond & $\mathrm{r}_{\mathrm{eq}}(\AA)$ & $\mathrm{K}_{\mathrm{r}}\left(\mathrm{kcal} / \mathrm{mol}^{2}\right)$ \\
\hline C1-H1 & 1.080 & 340.0 & CT-CT & 1.526 & 310.0 \\
CW-H4 & 1.070 & 385.0 & CT-C1 & 1.526 & 310.0 \\
CR-H5 & 1.070 & 380.0 & CT-HC & 1.090 & 340.0 \\
CW-NA & 1.378 & 360.0 & BF-FB & 1.410 & 290.0 \\
CR-NA & 1.325 & 400.0 & FB-FB & 2.303 & 290.0 \\
C1-NA & 1.472 & 280.0 & HW-HW & 1.5139 & 554.1 \\
CW-CW & 1.343 & 410.0 & OW-HW & 0.9572 & 554.1 \\
\hline
\end{tabular}


Table S3: Angle parameters.

\begin{tabular}{|c|c|c|c|c|c|}
\hline Angle & $\theta_{\mathrm{eq}}($ degree $)$ & $\mathrm{K}_{\theta}\left(\mathrm{kcal} / \mathrm{mol} \mathrm{radian}^{2}\right)$ & Angle & $\theta_{\mathrm{eq}}($ degree $)$ & $\mathrm{K}_{\theta}\left(\mathrm{kcal} / \mathrm{mol} \mathrm{radian}^{2}\right)$ \\
\hline H1-C1-H1 & 109.5 & 35.0 & CW-CW-H4 & 130.7 & 30.0 \\
H1-C1-NA & 109.5 & 55.0 & HC-CT-HC & 109.5 & 35.0 \\
C1-NA-CW & 125.7 & 50.0 & CT-CT-CT & 109.5 & 40.0 \\
C1-NA-CR & 126.3 & 50.0 & CT-CT-HC & 109.5 & 50.0 \\
CW-CW-NA & 107.1 & 120.0 & CT-CT-C1 & 109.5 & 40.0 \\
CR-NA-CW & 108.0 & 120.0 & CT-C1-H1 & 109.5 & 50.0 \\
NA-CR-NA & 109.9 & 120.0 & C1-CT-HC & 109.5 & 50.0 \\
H4-CW-NA & 122.1 & 30.0 & CT-C1-NA & 109.5 & 55.0 \\
H5-CR-NA & 125.7 & 30.0 & & & \\
\hline
\end{tabular}

Table S4: Dihedral and improper torsion parameters.

\begin{tabular}{|c|c|c|c|c|c|c|c|}
\hline Dihedral & $\mathrm{V}_{\mathrm{n}} / 2(\mathrm{kcal} / \mathrm{mol})$ & $\gamma$ & $\mathrm{n}$ & Dihedral & $\mathrm{V}_{\mathrm{n}} / 2(\mathrm{kcal} / \mathrm{mol})$ & $\gamma$ & $\mathrm{n}$ \\
\hline NA-CR-NA-CW & 12.0 & 180.0 & 2 & CT-C1-NA-CR & 0.16 & 0 & 3 \\
NA-CR-NA-C1 & 2.0 & 180.0 & 2 & H1-C1-CT-CT & 0.16 & 0 & 3 \\
H5-CR-NA-CW & 1.5 & 180.0 & 2 & NA-C1-CT-CT & 0.16 & 0 & 3 \\
H5-CR-NA-C1 & 1.5 & 180.0 & 2 & HC-CT-C1-H1 & 0.15 & 0 & 3 \\
CW-CW-NA-CR & 12.0 & 180.0 & 2 & HC-CT-C1-NA & 0.15 & 0 & 3 \\
CW-CW-NA-C1 & 2.0 & 180.0 & 2 & HC-CT-CT-HC & 0.15 & 0 & 3 \\
H4-CW-NA-CR & 2.0 & 180.0 & 2 & HC-CT-CT-CT & 0.16 & 0 & 3 \\
H4-CW-NA-C1 & 1.5 & 180.0 & 2 & CT-CT-CT-C1 & 0.18 & 0 & 3 \\
NA-CW-CW-H4 & 1.5 & 180.0 & 2 & CT-CT-CT-C1 & 0.25 & 180.0 & 2 \\
NA-CW-CW-NA & 12.0 & 180.0 & 2 & CT-CT-CT-C1 & 0.20 & 180.0 & 1 \\
H4-CW-CW-H4 & 1.5 & 180.0 & 2 & HC-CT-CT-C1 & 0.16 & 0 & 3 \\
H1-C1-NA-CW & 0.24 & 0 & 3 & CR-CW-NA-C1 & 2.00 & 180.0 & 2 \\
H1-C1-NA-CR & 0.16 & 0 & 3 & NA-NA-CR-H5 & 1.10 & 180.0 & 2 \\
CT-C1-NA-CW & 0.24 & 0 & 3 & CW-NA-CW-H4 & 1.10 & 180.0 & 2 \\
\hline
\end{tabular}



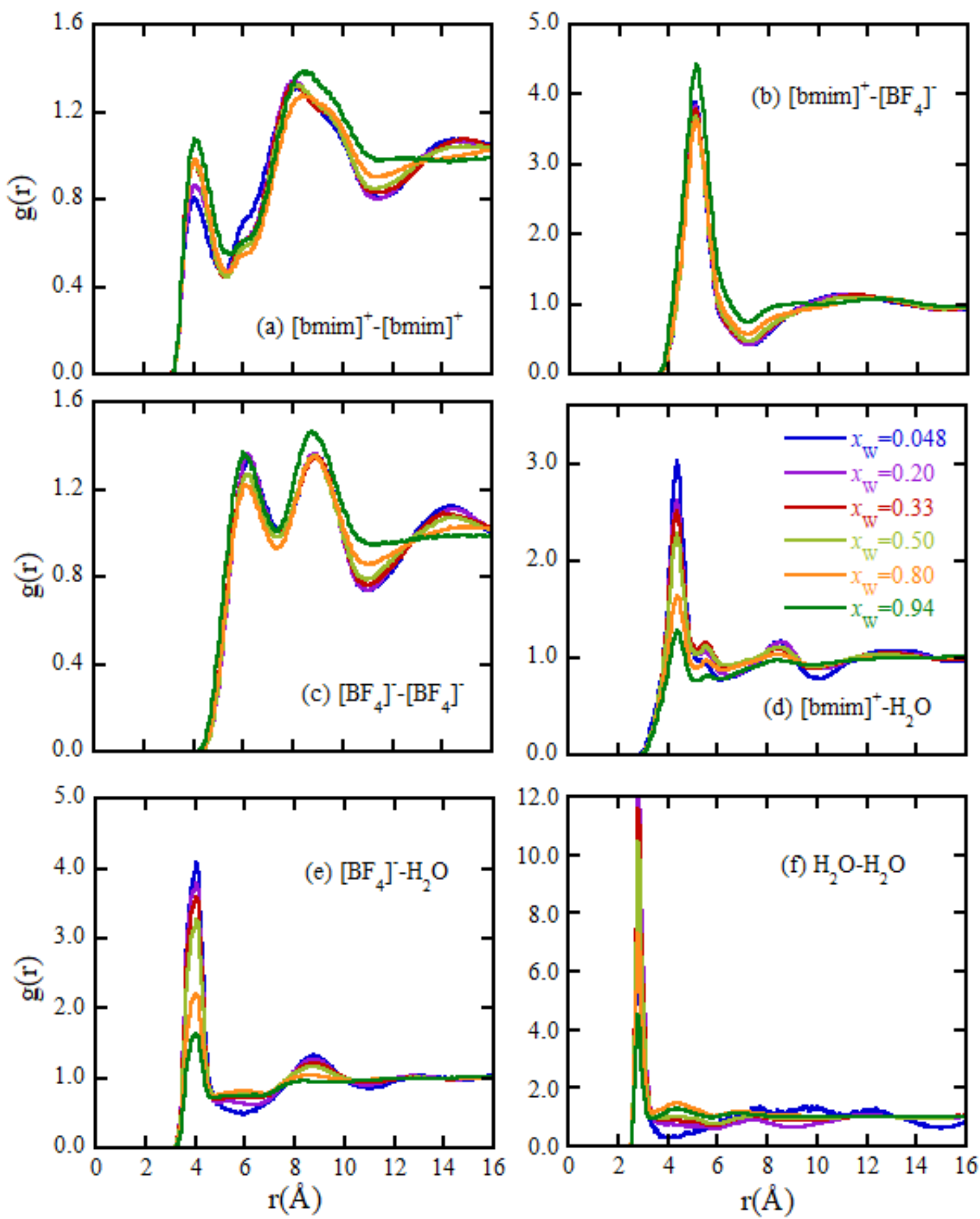

Figure S1. Calculated radial distribution functions of (a) $[\mathrm{bmim}]^{+}-[\mathrm{bmim}]^{+}$, (b) $[\mathrm{bmim}]^{+}-\left[\mathrm{BF}_{4}\right]^{-}$, (c) $\left[\mathrm{BF}_{4}\right]^{-}$ $-\left[\mathrm{BF}_{4}\right]^{-}$, (d) $[\mathrm{bmim}]^{+}-\mathrm{H}_{2} \mathrm{O}$, (e) $\left[\mathrm{BF}_{4}\right]^{-}-\mathrm{H}_{2} \mathrm{O}$, and (f) $\mathrm{H}_{2} \mathrm{O}-\mathrm{H}_{2} \mathrm{O}$ at several water mole fractions. Here, the center-of-mass of the imidazolium ring is used to present $[\mathrm{bmim}]^{+}$and the center-of-mass is used to present $\left[\mathrm{BF}_{4}\right]^{-}$and $\mathrm{H}_{2} \mathrm{O}$. 


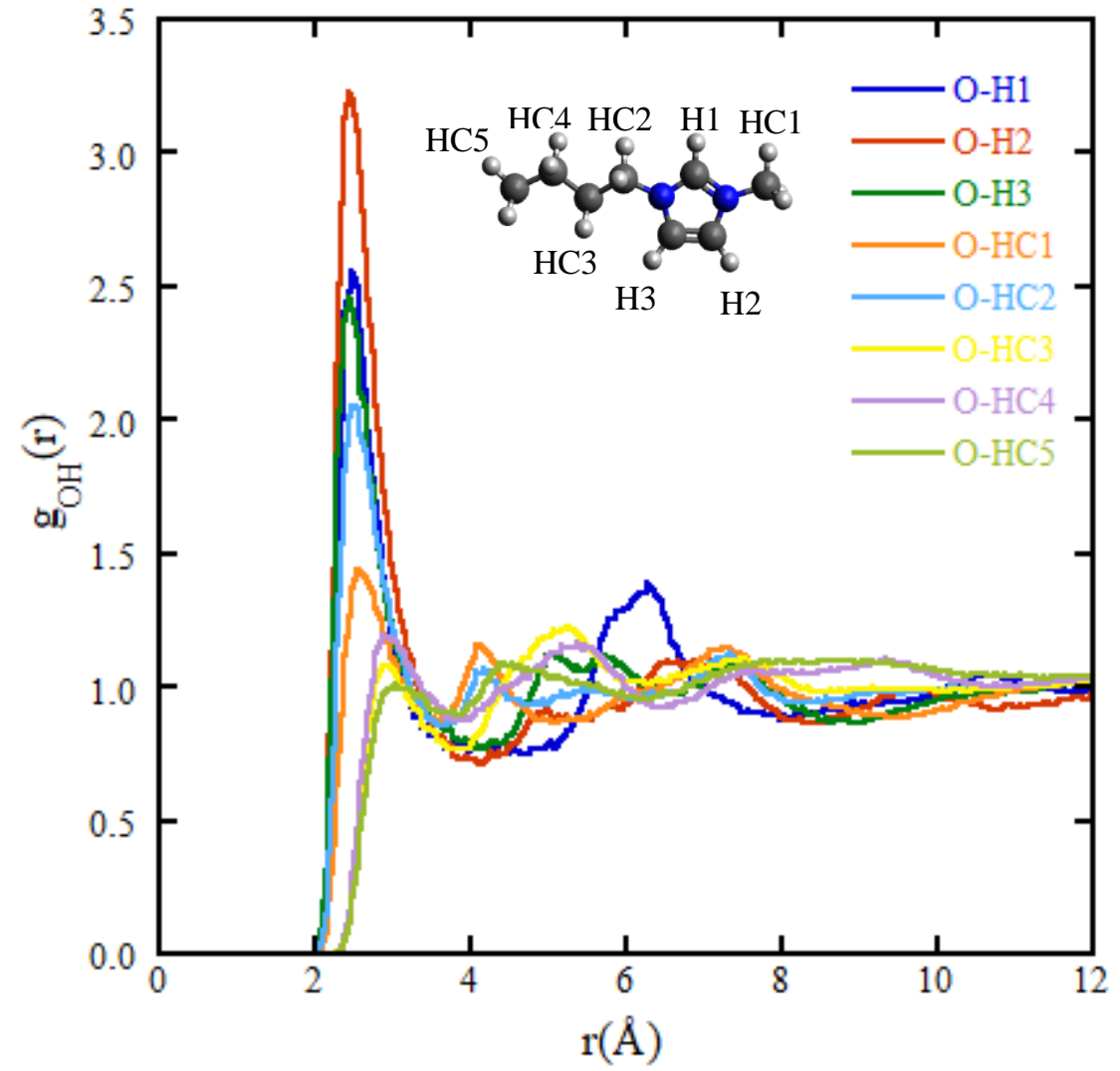

Figure S2. Atomic pair correlation functions, $\mathrm{g}_{\mathrm{OH}}(\mathrm{r})$, between $\mathrm{H}_{2} \mathrm{O}-\mathrm{O}$ and $[\mathrm{bmim}]^{+}-\mathrm{H}$ at $x_{\mathrm{W}}=0.048$. 

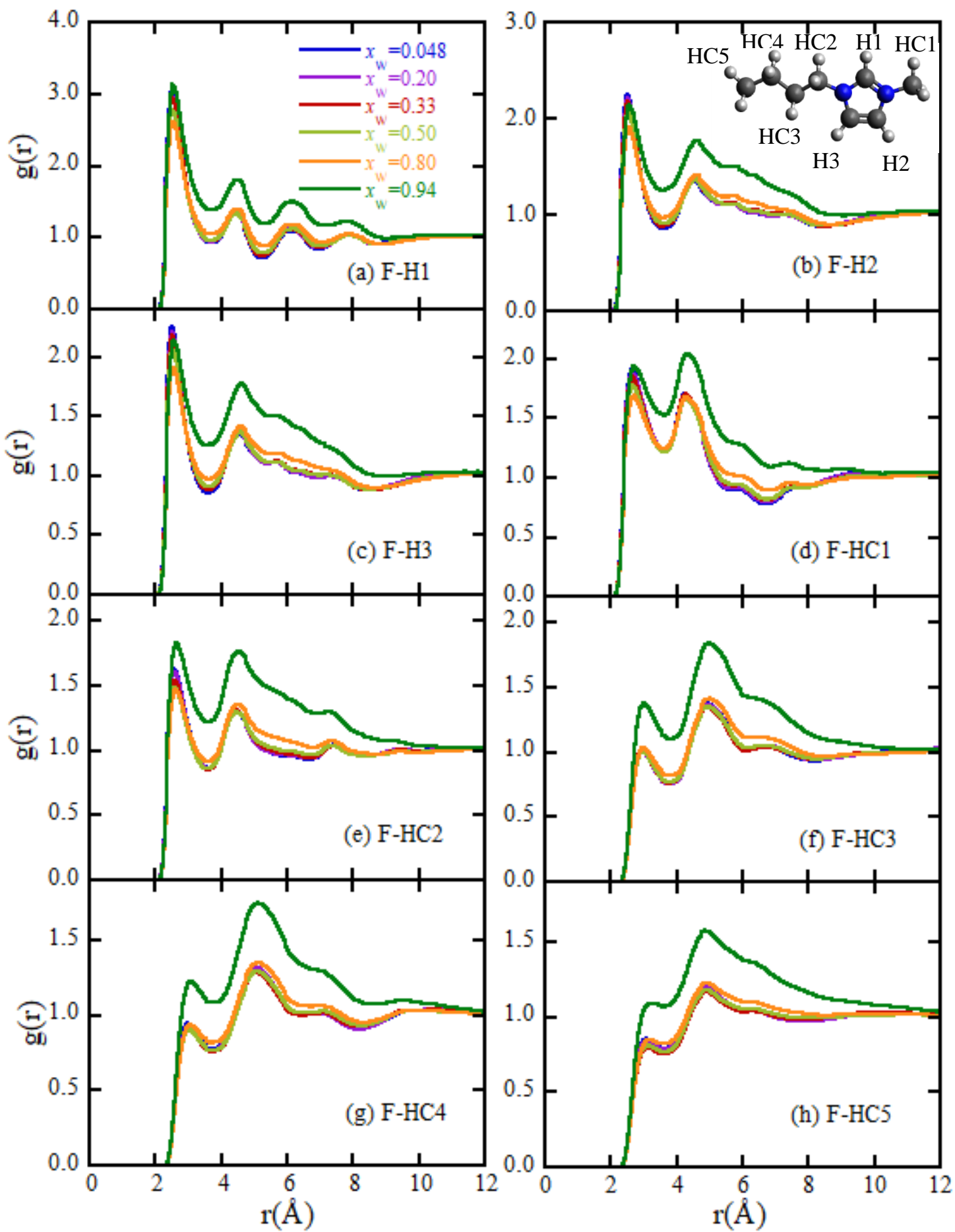

Figure S3. Decomposed atomic pair correlation functions, $g(r)$, between $\mathrm{F}$ of $\left[\mathrm{BF}_{4}\right]^{-}$and different $\mathrm{H}$ atoms of [bmim $]^{+}$cations (see figure for the $\mathrm{H}$ labels) at several water mole fractions. 

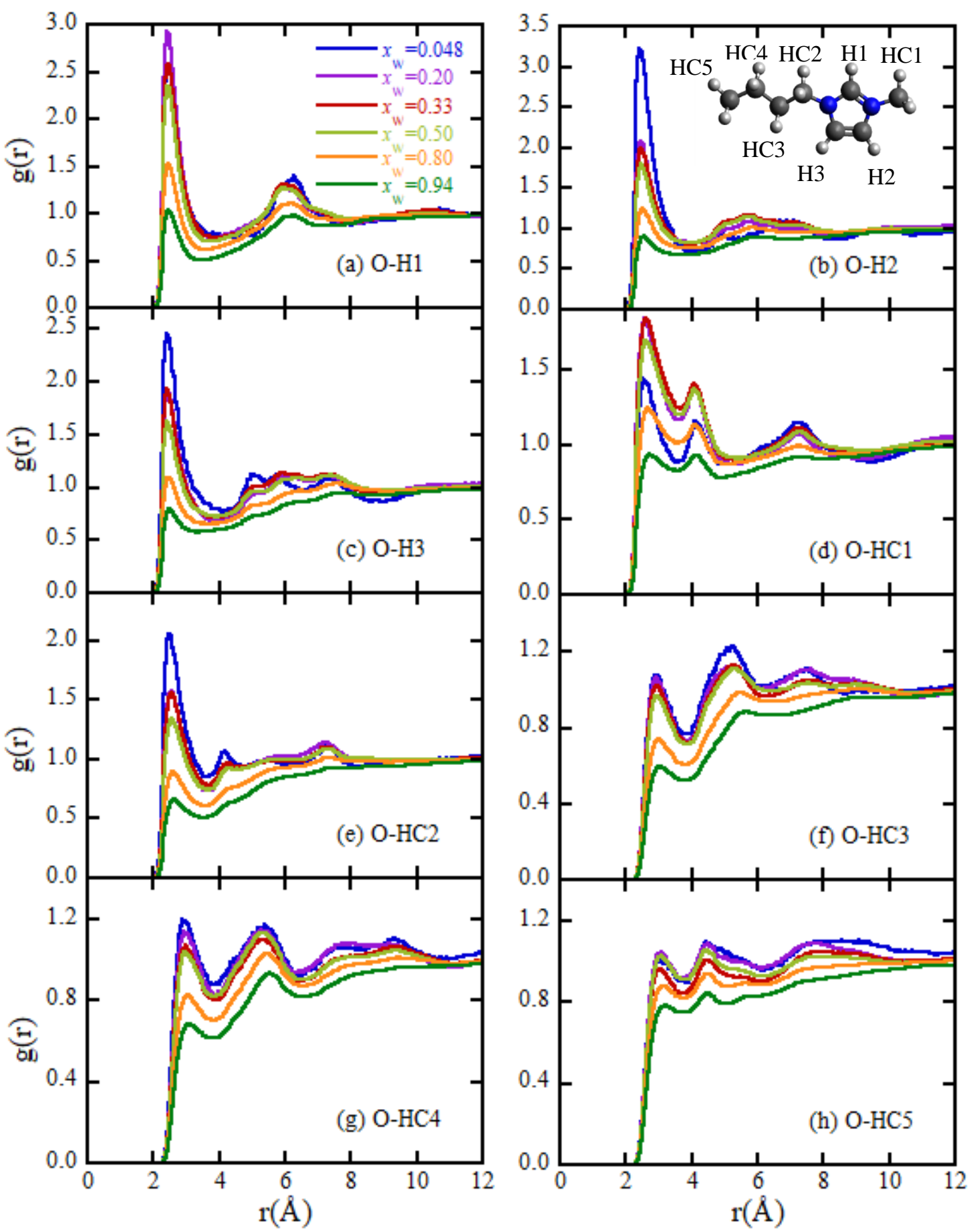

Figure S4. Decomposed atomic pair correlation functions, $g(r)$, between $\mathrm{O}$ of $\mathrm{H}_{2} \mathrm{O}$ and different $\mathrm{H}$ atoms of $[\mathrm{bmim}]^{+}$cations (see figure for the $\mathrm{H}$ labels) at several water mole fractions. 


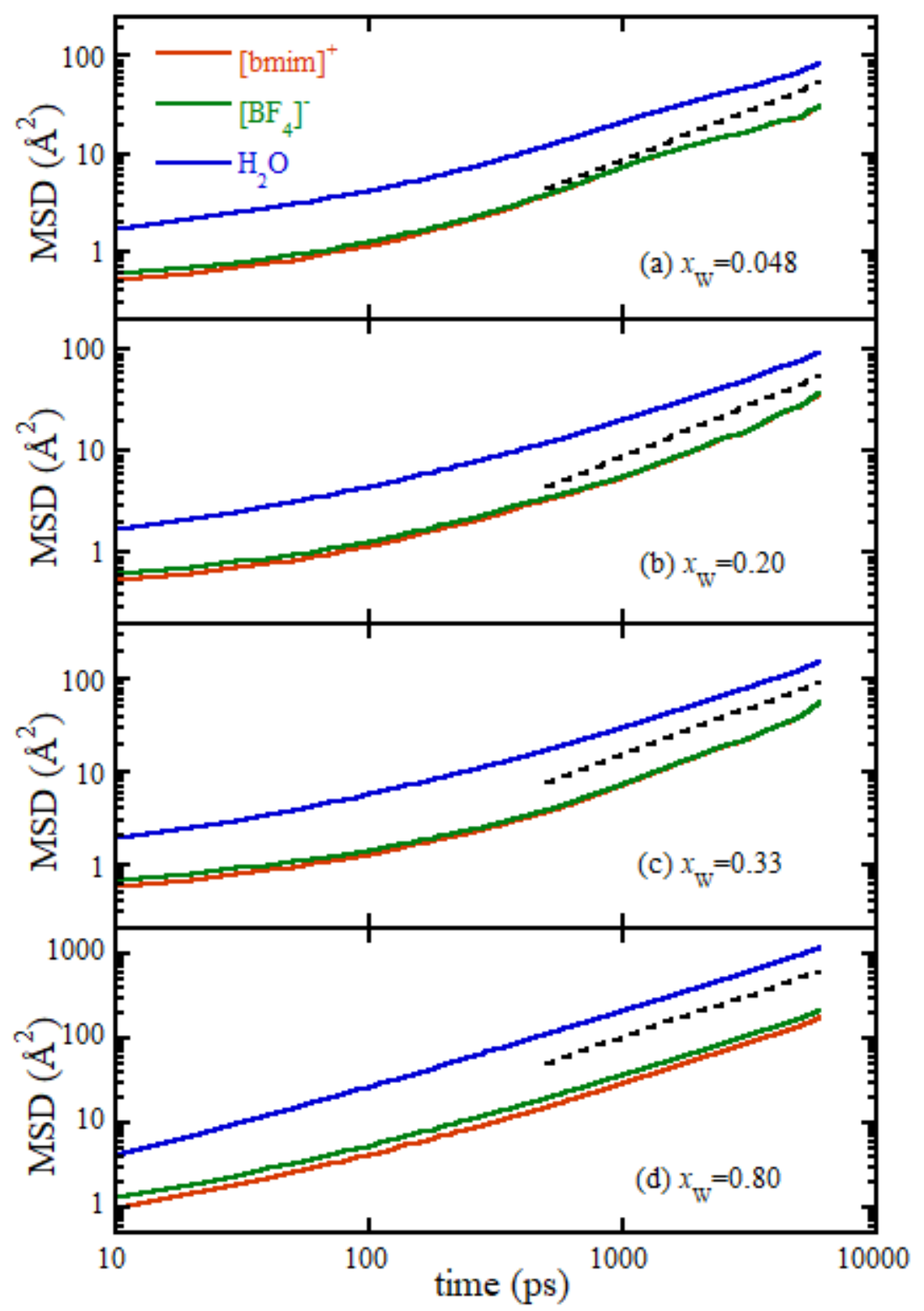

Figure S5. The computed mean square displacements (MSD) of $[\mathrm{bmim}]^{+},\left[\mathrm{BF}_{4}\right]^{-}$, and $\mathrm{H}_{2} \mathrm{O}$ as a function of time in log-log scale at water mole fractions of (a) $x_{\mathrm{W}}=0.048$, (b) $x_{\mathrm{W}}=0.20$, (c) $x_{\mathrm{W}}=0.33$, and (d) $x_{\mathrm{W}}=0.80$. The dark dashed line has a slope of 1 , corresponding to the diffusion limit. 
Table S5 Fitting data for ring normal of [bmim $]^{+}$

\begin{tabular}{|c|c|c|c|c|c|}
\hline$x_{\mathrm{W}}$ & $c_{0}$ & $c_{1}$ & $\tau_{1}(\mathrm{ps})$ & $c_{2}$ & $\tau_{2}(\mathrm{ps})$ \\
\hline 0.048 & 0.560 & 0.076 & 60.25 & 0.244 & 1527 \\
\hline 0.20 & 0.521 & 0.087 & 58.57 & 0.268 & 1353 \\
\hline 0.33 & 0.529 & 0.086 & 43.61 & 0.266 & 1005 \\
\hline 0.50 & 0.056 & 0.275 & 40.52 & 0.505 & 402.2 \\
\hline 0.80 & 0.228 & .0175 & 10.20 & 0.492 & 152.4 \\
\hline 0.94 & 0.048 & 0.345 & 9.78 & 0.485 & 85.0 \\
\hline
\end{tabular}

Table S6 Fitting data for C-N vector of [bmim $]^{+}$

\begin{tabular}{|c|c|c|c|c|c|}
\hline$x_{\mathrm{W}}$ & $c_{0}$ & $c_{1}$ & $\tau_{1}(\mathrm{ps})$ & $c_{2}$ & $\tau_{2}(\mathrm{ps})$ \\
\hline 0.048 & 0.551 & 0.108 & 116 & 0.251 & 2219 \\
\hline 0.20 & 0.544 & 0.104 & 84.7 & 0.266 & 1893 \\
\hline 0.33 & 0.535 & 0.100 & 46.4 & 0.288 & 953.9 \\
\hline 0.50 & 0.389 & .0125 & 55.4 & 0.405 & 858.2 \\
\hline 0.80 & 0.144 & 0.163 & 25.7 & 0.620 & 265.2 \\
\hline 0.94 & 0.127 & 0.263 & 22.6 & 0.650 & 119.8 \\
\hline
\end{tabular}

